\title{
RESISTÊNCIA À FERRUGEM DA FOLHA E POTENCIAL PRODUTIVO EM GENÓTIPOS DE TRIGO $\left({ }^{1}\right)$
}

\author{
JOÃO CARLOS FELICIO $\left({ }^{2 *}\right)$; CARLOS EDUARDO DE OLIVEIRA CAMARGO $\left({ }^{2,5}\right)$; \\ MÁRCIA SOARES CHAVES $\left({ }^{3}\right)$; JAIRO LOPES DE CASTRO $\left({ }^{4}\right)$
}

\begin{abstract}
RESUMO
Foram avaliados 18 genótipos de trigo provenientes de cruzamentos entre a linhagem BH1146//AA"S" / WIN"S", oriunda de cruzamentos interespecíficos entre Triticum aestivum L. e Triticum durum L., e as cultivares IAC 289 e IAC 1004, em experimentos instalados em condições de sequeiro em Manduri (Zona A); Capão Bonito e Itapeva (Zona B) e Paranapanema (Zona C), no período de 2003 a 2005. As avaliações dos genótipos ao agente causal da ferrugem da folha foram feitas no estádio de plântulas em casa de vegetação, onde os genótipos foram submetidos à inoculação, individualmente, com esporos de 12 raças de Puccinia triticina, as quais representavam o espectro de virulência do patógeno ocorrente no Brasil e sob infecção natural no campo. Foram avaliados os rendimentos de grãos de cada genótipo nas diferentes regiões e no conjunto dos experimentos, bem como a estabilidade e adaptabilidade. Os genótipos 8 (BH1146//AA"S"/WIN"S"/3/BUC/FKL//MYNA/VUL), 12 e 14 (BH1146// AA"S" /WIN"S" /3/VEE//DOVE/BUC) destacaram-se por sua resistência às raças fisiológicas de Puccinia triticina em casa de vegetação na fase de plântulas. Em condições de infecção natural da doença, destacaram-se os genótipos 4, 5, 8, 12, 13, 16 e 20 e a cultivar IAC 1004 (T. durum) com resistência à ferrugem da folha no triênio. Os melhores rendimentos de grãos foram obtidos pelos genótipos 8 (BH1146//AA"S" /WIN"S" /3/BUC/FKL// MYNA/VUL), 7 (BH1146//AA"S"/WIN"S"/3/HANN*2/PRL) e 18 (CMH 75.A.66/ SERI/3/BH1146//AA"S"/ WIN"S"). O menor foi obtido pelo genótipo 16 (KAUZ/3/ BH1146//AA"S" /WIN"S").
\end{abstract}

Palavras-chave: Triticum aestivum L. e Triticum durum L, resistência à ferrugem da folha, rendimento de grãos, estabilidade e adaptabilidade.

\section{ABSTRACT \\ LEAF RUST RESISTANCE AND GRAIN YIELD POTENTIAL IN WHEAT GENOTYPES}

Eighteen wheat genotypes obtained from crosses between the parental line BH1146//AA"S" / WIN"S" originated from interespecific crosses between Triticum aestivum L. and Triticum durum L. and the cultivars IAC 289 and IAC 1004 were evaluated in experiments carried out in upland conditions at Manduri (Zone A); Capão Bonito and Itapeva (Zone B) and Paranapanema (Zone C), in the State of São Paulo, Brazil, during 2003-2005 crop seasons. The evaluation of the genotypes to the causal agent of leaf rust was made at the seedling stage in greenhouse, where the genotypes were individually inoculated with spores of 12 races of Puccinia triticina, which represented the spectrum of pathogen virulence occurring in Brazil and under natural infection out in the field. Grain yield of each genotype was evaluated in the different regions and in a group of experiments, as well as the stability and adaptability. The genotypes 8 (BH1146// AA"S"/WIN"S" /3/BUC/FKL//MYNA/VUL), 12 and 14 (BH1146// AA"S" /WIN"S" /3/VEE / / DOVE/ BUC) showed resistance the physiologic races of Puccinia triticina in greenhouse in the seedling stage. The genotypes $4,5,8,12,13$, 16 e 20 and the cultivar IAC 1004 (T. durum) presented leaf rust resistance, under natural disease infection conditions. The highest grain yields were obtained by the genotypes 8 (BH1146// $\mathrm{AA}^{\prime \prime} \mathrm{S}^{\prime \prime} / \mathrm{WIN} \mathrm{S}^{\prime \prime} / 3 /$ BUC/FKL//MYNA/VUL), 7 (BH1146//AA"S" /WIN"S"/3/HANN²/ PRL) and 18 (CMH75.A.66/SERI/ 3/BH1146/ / AA"S"/WIN"S"). Genotype 16 (KAUZ/3/ BH1146/ /AA"S" /WIN"S") presented the lowest yield.

Key words: Triticum aestioum L. and Triticum durum L, resistance to leaf rust, yield stability and adaptability.

( ${ }^{1}$ ) Com apoio financeiro da FAPESP. Recebido para publicação em 9 de janeiro de 2007 e aceito em 27 de maio de 2008.

$\left({ }^{2}\right)$ Instituto Agronômico (IAC), Centro de Análise e Pesquisa Tecnológica dos Agronegócios de Grãos e Fibras, Caixa Postal 28, 13001-970 Campinas (SP). E-mail: jfelicio@iac.sp.gov.br $\left(^{*}\right)$ Autor correspondente; ccamargo@iac.sp.gov.br

(3) Embrapa Trigo, Caixa Postal 451, 99001-970 Passo Fundo (RS). E-mail mchaves@cnpt.embrapa.br

$\left({ }^{4}\right)$ Pólo Regional de Desenvolvimento Tecnológico dos Agronegócios do Sudoeste Paulista, APTA/DDD, Caixa Postal 6258, 8300-970 Capão Bonito (SP).

$\left({ }^{5}\right)$ Com bolsa de produtividade em pesquisa do CNPq. 


\section{INTRODUÇÃO}

Dentre as doenças que atacam a cultura do trigo (Triticum aestivum L.), a ferrugem da folha (Puccinia triticina Erikss., sin. Puccinia recondita Rob. ex Desm. f. sp. tritici) tem sido a mais destrutiva, ocorrendo em todas as regiões do mundo em que este cereal é cultivado. A ferrugem da folha, em alguns anos, ocorre já no estádio de plântula, mas geralmente ataca mais tarde, podendo prolongar-se até próximo à maturação dos grãos, provocando a secagem prematura das folhas. Em genótipos suscetíveis, a severidade pode atingir níveis elevados, comprometendo tanto o rendimento que pode sofrer reduções entre $30 \%$ e $50 \%$, quanto a qualidade dos grãos (Roelfs et al, 1992; ReIs et al., 1996; Picinini e FERNANDES, 2000).

A população patogênica de $P$ uccinia triticinia presente na região do Cone Sul da América do Sul é extremamente dinâmica, e no Brasil, a cada ano, têm surgido até três novas raças fisiológicas do patógeno (BArcellos, 1999, Chaves et al., 2005). O acompanhamento das alterações de virulência, nas regiões tritícolas do Brasil e do Cone Sul, é indispensável aos programas de melhoramento genético de trigo, visando à estabilidade da resistência, a qual tem sido limitada pela freqüente superação da resistência das cultivares melhoradas por novas virulências, na população do patógeno, assim como a falta de conhecimento sobre a movimentação dos esporos na região.

O uso de fungicidas é a forma mais comum de controlar a doença. Este método de controle, embora eficiente, tem grande impacto econômico e ambiental (GERMÁn et al., 2004). No entanto, a resistência genética também tem sido amplamente buscada em programas de melhoramento genético, uma vez que seu valor no controle de doenças de plantas é reconhecido há vários anos (Nelson, 1973; Priestley e BAyles, 1988). Os avanços na genética e as vantagens óbvias de plantar variedades resistentes tornaram o melhoramento para resistência possível e desejável, e grande progresso foi alcançado a partir da segunda metade do século XX (SOUSA, 2001; CUNHA, 2005).

Entretanto, o estreitamento na base genética do germoplasma atualmente disponível e a plasticidade da população patogênica de $P$. triticina têm levado a um cenário em que se verifica a ocorrência de ciclos sucessivos de superação de resistência e a conseqüente substituição de cultivares. Esses fatores implicam a necessidade constante de buscar novas fontes de resistência, de modo que aumentem as chances de sucesso desta alternativa de controle. Neste contexto, a introgressão de genes de espécies afins ao trigo cultivado representa uma alternativa com grandes potencialidades econômicas (BARbosa et al. 1993, Angra et al. 1996).

Linhagens de trigo comum (T. aestivum) oriundas de seleções em população submetida à irradiação gama, em geração $\mathrm{F}_{4}$, do cruzamento interespecífico entre a cultivar BH1146 (T. aestivum) e Anhinga"S" / Winged"S" (T. durum) destacaram-se quanto à produção de grãos e resistência ao agente da ferrugem da folha, em experimentos instalados em condição de sequeiro e de irrigação por aspersão no Estado de São Paulo (CAMARGO et al., 1995). O genótipo de trigo IAC 356, proveniente desse cruzamento foi avaliado no período de 1994 a 2001, no município de Capão Bonito, localizado na Região Sudoeste paulista, com resistência ao agente causal da ferrugem da folha (Felicio et al., 2004).

O objetivo deste trabalho foi avaliar o desempenho de 18 novos genótipos de trigo ( $T$. aestivum.) quanto à resistência à ferrugem da folha, ao rendimento de grãos, à adaptação e à estabilidade, quando comparados às cultivares IAC 289 ( $T$. aestivum) e IAC 1004 (T. durum).

\section{MATERIAL E MÉTODOS}

Foram semeados experimentos em diferentes regiões tritícolas do Estado de São Paulo no período de 2003 a 2005, em ambiente de sequeiro nas localidades de Manduri (Zona A), Capão Bonito e Itapeva (Zona B) e em Paranapanema (Zona C). Para a instalação dos experimentos, adotaram-se as recomendações da Comissão Técnica de Trigo da Secretaria Agricultura e do Abastecimento do Estado de São Paulo (CAmpinas, Instituto Agronômico, 2002). As informações quanto à genealogia, o ciclo em dias, da emergência ao início do espigamento (estádio de crescimento 10.1 na escala de LARGE, 1954), da emergência à maturação plena (estádio de crescimento 11.4 na escala de LARGE, 1954), e a altura das plantas estão relacionadas na tabela 1.

Os experimentos foram delineados em blocos ao acaso, compostos de vinte genótipos com quatro repetições por local. Cada parcela foi constituída de oito linhas de $3 \mathrm{~m}$ de comprimento, com espaçamento de $0,15 \mathrm{~m}$ entre elas, com separação lateral de $0,60 \mathrm{~m}$ entre as parcelas. Procedeu-se à semeadura com 80 sementes viáveis por metro de sulco, sendo efetuada a colheita da área total das parcelas.

A adubação mineral foi feita a lanço antes da semeadura e, posteriormente, incorporada ao solo. As quantidades de fertilizantes aplicadas nos diferentes locais foram definidas de acordo com as recomendações de adubação e calagem para o Estado de São Paulo ( Raij et al., 1996). 
Tabela 1. Genealogia dos genótipos, características agronômicas do ciclo em dias da emergência ao início do florescimento e da emergência à maturação plena e da altura do genótipo de trigo, avaliado em condições de sequeiro em Capão Bonito, Estado de São Paulo, de 2003 a 2005

\begin{tabular}{|c|c|c|c|}
\hline Genealogia & Glorescimento & Maturação & Altura \\
\hline & dias & dias & $\mathrm{cm}$ \\
\hline 1-BH1146// AA"S" / WIN"S"/3/IAC 227 & 59 & 118 & 81 \\
\hline 2-BH1146// AA"S" / WIN"S"/3/IAC 227 & 62 & 118 & 96 \\
\hline 3-BH1146// AA"S" /WIN"S" /6/IAS58/4/KAL/BB/CS"S" /3/ALD"S" /5/BOW"S" & 62 & 118 & 80 \\
\hline 4-BH1146/ / AA"S" /WIN"S" /3/IAC 24 & 64 & 123 & 82 \\
\hline 5-BH1146/ / AA"S" /WIN"S" /3/IAC 24 & 62 & 123 & 84 \\
\hline 6-BH1146// AA"S" /WIN"S" /3/TUI"S" & 59 & 118 & 81 \\
\hline 7-BH1146//AA"S" /WIN"S"/3/HAHN*2/PRL & 62 & 118 & 87 \\
\hline 8-BH1146//AA"S" /WIN"S"/3/BUC/FKL//MYNA/VUL & 62 & 118 & 84 \\
\hline 9-BH1146/ / AA"S" /WIN"S" / 4/INIA/A.DIST//INIA/3/GEN & 59 & 118 & 81 \\
\hline 10-IAC 1004 (T.durum L.) & 62 & 125 & 89 \\
\hline 11-BH1146/ / AA"S" / WIN"S" /3/SERI 82 & 59 & 120 & 92 \\
\hline 12-BH1146// AA"S" / WIN"S"/3/VEE//DOVE/BUC & 59 & 120 & 77 \\
\hline 13-BH1146//AA"S" / WIN"S" /3/CNO79/PRL & 62 & 123 & 86 \\
\hline 14-BH1146// AA"S" /WIN"S"/3/VEE/ / DOVE/BUC & 55 & 118 & 90 \\
\hline 15-IAC 289 (T.aestivum L.) & 64 & 125 & 89 \\
\hline 16-KAUZ/3/BH 1146/ / AA"S" / WIN"S" & 64 & 130 & 86 \\
\hline 17-VEE//DOVE/BUC/3/BH1146// AA"S" /WIN"S" & 64 & 123 & 100 \\
\hline 18-CMH 75.A.66/SERI/3/BH1146/ / AA"S" / WIN"S" & 64 & 123 & 93 \\
\hline 19-BH1146// AA"S" /WIN"S"/3/TAN/PEW//SARA & 59 & 123 & 88 \\
\hline 20-BH1146//AA"S" /WIN"S" / 4/RL6010/2*YR/3/2*VEE"S" / GEN & 59 & 120 & 80 \\
\hline
\end{tabular}

O controle de pragas foi realizado com a aplicação de inseticidas de acordo com as recomendações da Comissão Técnica de Trigo da Secretaria Agricultura e do Abastecimento do Estado de São Paulo (Campinas, Instituto Agronômico, 2002).

As avaliações para resistência à ferrugem da folha foram realizadas em dois estádios:

No estádio de planta adulta, a ferrugem-dafolha (Puccinia triticina Rob.ex-Desm. f.sp. tritici Erichs) foi avaliada, sob condições de infecção natural após o florescimento das plantas (estádio de crescimento 11.2 na escala de LARGE, 1954), por meio da escala modificada de Cobb, conforme Schramm et al. (1974). Essa escala vai de 0 a $99 \%$ de área foliar infectada, complementada pelo tipo de reação: $\mathbf{S}$ = suscetível (uredossoro grande, coalescente, sem necrose e sem clorose); MS = moderadamente suscetível (uredossoro médio, que pode ou não estar associado com clorose); MR = moderadamente resistente (uredossoro pequeno a médio, freqüentemente circundado por clorose ou necrose); $\mathbf{R}$ resistente (uredossoro minúsculo, rodeado de áreas necróticas ou cloróticas).

Em casa de vegetação, no estádio de plântulas foram submetidas à inoculação, individualmente, 12 raças de Puccinia triticina, as quais representam o espectro de virulência do patógeno ocorrente no Brasil, em condições controladas da Embrapa Trigo, Passo Fundo (RS). As raças foram provenientes do banco de raças do patógeno, mantido por essa instituição. Cada raça corresponde a uma combinação de avirulência/ virulência, com possíveis variações em função de genes sensíveis à temperatura, e cada combinação de virulência é representada por um código, de acordo com o Sistema Norte-Americano de Nomenclatura (Long e Kolmer, 1989). No Brasil, estas combinações de virulência são também designadas pela letra $B$, seguido pelo número de ordem de identificação de cada raça no país (Tabela 2 ). 
Tabela 2. Porcentagem de área foliar infectada em condições natural no campo pelo agente causal da ferrugem da folha, (Puccinia triticina) nos genótipos de trigo avaliados em condição de sequeiro, nas localidades de Manduri (Zona A), Capão Bonito e Itapeva (Zona B) e em Paranapanema (Zona C), no Estado de São Paulo, no período de 2003 a 2005

\begin{tabular}{|c|c|c|c|c|c|c|c|c|c|c|c|c|c|}
\hline \multirow{2}{*}{ Genótipo } & \multicolumn{3}{|c|}{ Manduri } & \multicolumn{3}{|c|}{ Capão Bonito } & \multicolumn{3}{|c|}{ Itapeva } & \multicolumn{3}{|c|}{ Paranapanema } & \multirow{2}{*}{ Reação } \\
\hline & 2003 & 2004 & 2005 & 2003 & 2004 & 2005 & 2003 & 2004 & 2005 & 2003 & 2004 & 2005 & \\
\hline 1 & $20 \mathrm{~S}$ & TS & 0 & 0 & $5 S$ & $5 S$ & $20 S$ & $10 \mathrm{~S}$ & TS & $50 S$ & $20 S$ & $5 S$ & S \\
\hline 2 & $10 \mathrm{~S}$ & TS & $20 S$ & 0 & $30 \mathrm{~S}$ & $10 \mathrm{~S}$ & $20 \mathrm{~S}$ & $20 S$ & TS & $50 S$ & $20 S$ & $5 S$ & $\mathrm{~S}$ \\
\hline 3 & $5 \mathrm{MS}$ & 0 & 0 & 0 & $10 \mathrm{~S}$ & 0 & 0 & 0 & 0 & 0 & 0 & 0 & MS \\
\hline 4 & 10MR & 0 & 0 & 0 & 0 & 0 & 0 & 0 & 0 & 0 & 0 & 0 & MR \\
\hline 5 & TR & 0 & 0 & 0 & $5 S$ & 0 & 0 & 0 & 0 & $10 \mathrm{MS}$ & 0 & 0 & MS \\
\hline 6 & $5 \mathrm{MR}$ & 0 & $10 \mathrm{~S}$ & 0 & $5 S$ & 0 & 0 & $10 \mathrm{~S}$ & 0 & 5 & $40 \mathrm{~S}$ & 0 & S \\
\hline 7 & $5 \mathrm{MR}$ & 0 & 0 & 0 & $20 S$ & 0 & 0 & $5 \mathrm{MS}$ & 0 & TS & 0 & 0 & S \\
\hline 8 & $5 \mathrm{MR}$ & 0 & 0 & 0 & 0 & & 0 & 0 & $5 \mathrm{MS}$ & 0 & 10MS & 0 & MS \\
\hline 9 & $5 \mathrm{MR}$ & 0 & 0 & 0 & $20 S$ & $10 \mathrm{~S}$ & $5 S$ & $10 \mathrm{~S}$ & 0 & 0 & $40 \mathrm{~S}$ & 0 & S \\
\hline 10 & 0 & 0 & 0 & 0 & 0 & 0 & 0 & 0 & 0 & 0 & 0 & 0 & $\mathrm{R}$ \\
\hline 11 & $0-5 R$ & 0 & 0 & 0 & $20 \mathrm{~S}$ & $5 S$ & 0 & 0 & 0 & $10 \mathrm{~S}$ & 20MS & TS & S \\
\hline 12 & 0 & 0 & 0 & 0 & 0 & $5 S$ & 0 & 0 & 0 & 0 & 0 & 0 & MS \\
\hline 13 & 0 & TS & 0 & 0 & 0 & 0 & 0 & 0 & 0 & TS & $5 S$ & 0 & MS \\
\hline 14 & $10 \mathrm{~S}$ & TS & 0 & 0 & $10 \mathrm{~S}$ & 0 & 0 & 0 & 0 & $10 \mathrm{MS}$ & $5 S$ & 0 & $\mathrm{~S}$ \\
\hline 15 & $10 \mathrm{MS}$ & 0 & 0 & 0 & 0 & $10 \mathrm{~S}$ & 0 & 0 & 0 & $10 \mathrm{~S}$ & $30 S$ & 0 & S \\
\hline 16 & 0 & 0 & 0 & 0 & 0 & $5 \mathrm{MR}$ & 0 & $5 \mathrm{MR}$ & 0 & 0 & 0 & 0 & MR \\
\hline 17 & $10 \mathrm{MR}$ & 0 & 0 & 0 & $10 \mathrm{~S}$ & 0 & 0 & 0 & $10 \mathrm{~S}$ & $10 \mathrm{MR}$ & 10MR & 0 & S \\
\hline 18 & $10 \mathrm{MR}$ & 0 & 0 & 0 & 0 & 0 & 0 & 0 & 0 & 0 & 10MR & 0 & MR \\
\hline 19 & $20 S$ & 0 & $20 \mathrm{~S}$ & 0 & $20 S$ & $10 S$ & $10 S$ & $30 S$ & 0 & 0 & 10MR & $5 S$ & $S$ \\
\hline 20 & 0 & 0 & 0 & 0 & $5 S$ & 0 & 0 & 0 & 0 & $10 \mathrm{~S}$ & 10MR & 0 & MS \\
\hline
\end{tabular}

$\mathrm{S}=$ Suscetível. MS = Moderadamente suscetível. MR = Moderadamente resistente.

A inoculação foi realizada com os uredosporos do fungo resultantes da multiplicação de inóculo. Foi feita uma suspensão dos esporos em óleo mineral, com concentração de $10^{5}$ a $10^{6}$ esporos $\mathrm{mL}^{-1}$, a qual foi aspergida sobre as plântulas dos genótipos a serem avaliados, 7-9 dias após a emergência, quando a primeira folha estava completamente expandida. Após a inoculação, as plântulas foram incubadas em câmara úmida, no escuro, por 12 horas. Após o período de incubação, as plântulas foram transferidas para casa de vegetação totalmente isolada do ambiente externo, com condições controladas de temperatura (22 $+2{ }^{\circ} \mathrm{C}$ ) e umidade $(80 \%)$ até o aparecimento dos sintomas.

O aparecimento dos sintomas ocorreu em 10 a 12 dias após a inoculação, e após, procedeu-se à avaliação da reação dos genótipos, observando-se o tipo de infecção correspondente ao sintoma, de acordo com Roelfs et al. (1992). Foram considerados resistentes os genótipos com reação 0 , ;, 1 e 2 (e suas variações para mais ou menos) e suscetíveis aqueles com tipo de infecção 3 e 4 (e suas variações para mais ou menos).
O ciclo das cultivares foi determinado pelo número de dias decorridos da emergência das plântulas até a maturação. As cultivares com ciclo da emergência à maturação de 110 a 120,121 a 130 e acima de 130 dias foram consideradas, respectivamente, de ciclo precoce, médio e tardio.

A altura das plantas foi medida em centímetros no campo, na época da maturação, do nível do solo ao ápice da espiga, com exclusão das aristas, estimando-se a média de diferentes pontos de cada parcela.

A avaliação de rendimentos de grãos foi realizada pesando, em gramas, a produção total de cada parcela, a qual foi extrapolada para quilogramas por hectare.

Os experimentos foram analisados conjuntamente, avaliando-se efeitos de ano, local, genótipo e das interações ano $\mathrm{x}$ local, ano $\times$ genótipo, local $x$ genótipo e ano $x$ local $x$ genótipo. Utilizou-se para a comparação das médias o teste de Duncan, de acordo com Pimentel-Gomes (1990). 
Foram determinadas a estabilidade e a adaptabilidade pelo método proposto por EBERHART e Russell (1966), dos genótipos por região, bem como em todas as regiões em conjunto, com base nos coeficientes de cada genótipo em relação ao índice ambiental. Considerou-se como genótipo ideal aquele com alto rendimento médio de grãos, coeficiente de regressão igual a $1,0(b=1)$ e desvios da regressão igual a zero $\left(S^{2} \mathrm{~d}=0\right)$.

\section{RESULTADOS E DISCUSSÃO}

Os dados médios do ciclo da emergência ao início do espigamento e da emergência á maturação plena, expressos na tabela 1, caracterizaram o genótipo 16 (KAUZ/3/BH1146/ / AA"S" / WIN"S") como de ciclo tardio sendo os demais genótipos de ciclo médio e o porte do genótipo 17 (VEE//DOVE/ BUC/3/BH1146// AA"S" / WIN"S") como o mais alto $(100 \mathrm{~cm})$, os genótipos 2, 11 e 18 também são considerados de porte alto (maiores que $90 \mathrm{~cm}$ ).
Segundo Barcellos (1986), tem sido evidentemente comprovado que o aumento da área cultivada com determinada cultivar exerce uma pressão de seleção sobre a população patogênica, favorecendo a ocorrência de novas raças que podem tornar extremamente suscetível cultivares resistentes a todas as raças de Puccinia triticina. Com base nesta informação, é possível que alterações possam ocorrer futuramente nos genótipos, de acordo com as reações apresentadas entre as avaliações em plântula e planta adulta.

No período abrangido pela instalação dos experimentos, houve grande variação na ocorrência das raças, não só na sua freqüência como também no aparecimento de novas raças em infecções natural no campo. Considerando-se os genótipos no estádio de planta adulta (Tabela 2), foi possível classificar entre as melhores os genótipos 4, 5, 8, 12, 13, 16 e 20 como resistentes à ferrugem da folha, pela não-ocorrência de níveis elevados de infecção, no decorrer do triênio, e a cultivar IAC 1004 (Triticum durum), imune à doença.

Tabela 3. Tipo de infecção de genótipos de trigo a 12 raças de Puccinia triticina as quais representam o espectro de virulência do patógeno no Brasil em condições controlada em casa de vegetação

\begin{tabular}{lcccccccccccc}
\hline Gentótipo & B 35 & B 37 & B 38 & B 39 & B 40 & B 41 & B 43 & B 45 & B 48 & B 49 & B 50 & B 51 \\
\hline 1 & 3 & 3 & $2-3(\mathrm{pu})$ & 3 & 3 & $;$ & $2+3$ & 3 & 3 & 3 & 3 & 3 \\
2 & 3 & 3 & $2-3(\mathrm{pu})$ & 3 & 3 & 12 & $2+3$ & 3 & $3-$ & 3 & 3 & 3 \\
3 & 2 & 0 & $;$ & $0 ;$ & 32 & 2 & $0 ;$ & 3 & $3-$ & $;$ & 3 & 2 \\
4 & 3 & 3 & 3 & 3 & 3 & 3 & 3 & 3 & 3 & 3 & 3 & 3 \\
5 & $33-$ & 3 & 3 & 3 & 3 & 3 & 3 & 3 & 3 & 3 & 3 & 3 \\
6 & 2 & $;$ & $123(1 \mathrm{P})$ & $0 ;$ & $3-2+$ & 1 & $;$ & 3 & 3 & 3 & 3 & 3 \\
7 & 3 & 3 & $2-3$ & $3-2$ & 3 & $; 2(\mathrm{pu})$ & 23 & $3-$ & 3 & $21 ;$ & 3 & 3 \\
8 & $;$ & $0 ;$ & $3-2$ & 1 & $;$ & $0 ;$ & 3 & $0 ;$ & $22+3-$ & $0 ;$ & 3 & $;$ \\
9 & 12 & 3 & $3-$ & 3 & 21 & 3 & 3 & 3 & 3 & 3 & $2+3$ & $123(1 \mathrm{P})$ \\
10 & $32(\mathrm{pu})$ & 3 & $;$ & $23=$ & 23 & $; 1$ & 3 & 3 & $3-(4 \mathrm{P}) 1(2 \mathrm{P})$ & $3-$ & 23 & 23 \\
11 & 3 & 3 & 3 & 3 & $3-2$ & $;(6 \mathrm{P}) 3(2 \mathrm{p})$ & 3 & 3 & $33-$ & 3 & 3 & 3 \\
12 & 1 & $0 ;(31 \mathrm{P})$ & $;$ & 0 & $2-1$ & $;$ & 0 & $03(\mathrm{pu})$ & 21 & $; 1$ & 32 & $2+$ \\
13 & 3 & 3 & 3 & 3 & 3 & 3 & 3 & $3-$ & 3 & 3 & - & 3 \\
14 & $2+$ & $0 ; 3(1 \mathrm{P})$ & $;$ & $0 ;$ & 1 & $;$ & - & $23-$ & 21 & $; 1$ & 3 & $2+$ \\
15 & 3 & $3(5 \mathrm{P}) 1 ;(3 \mathrm{p})$ & $23-$ & 3 & $3-$ & $; 13(1 \mathrm{P})$ & 3 & $23-$ & $23(\mathrm{pu})$ & 3 & 3 & 3 \\
16 & 3 & $3 ;(1 \mathrm{P})$ & 3 & $3-$ & $3-$ & 3 & 3 & 3 & $3-$ & 3 & 3 & 3 \\
17 & 3 & 3 & 3 & $3-$ & $3-$ & 3 & 3 & 3 & 3 & 3 & 3 & 3 \\
18 & 3 & 3 & 3 & 3 & $3 ;(1 \mathrm{P})$ & 3 & 3 & 3 & 3 & 3 & 3 & 3 \\
19 & $3-$ & 3 & 3 & 0 & 3 & 3 & 3 & $0 ;$ & 3 & 3 & 3 & 3 \\
20 & $3-$ & 3 & $12(\mathrm{pu})$ & 3 & 3 & 1 & 3 & 3 & $;(5 \mathrm{P}) 3(3 \mathrm{P})$ & 3 & 3 & 3 \\
\hline
\end{tabular}

Resistência: $0, ;, 1,1+, 2,2+(\mathrm{pu})=$ pústulas

Suscetibilidade: $3,3-, 3=(\mathrm{P})=$ planta 
Os genótipos 1 e 2 provenientes do cruzamento BH 1146//AA"S" / WIN"S" / / IAC 227 caracterizaram-se pela maior suscetibilidade ao agente causal da ferrugem da folha.

Verificou-se pelo tipo de infecção (Tabela 3) que os genótipos 8 (BH1146//AA"S"/WIN"S"/3/ BUC/FKL//MYNA/VUL), 12 e 14 (BH1146//AA"S" / WIN"S" /3/VEE//DOVE/BUC) se destacaram por sua resistência às raças fisiológicas de Puccinia triticina em casa de vegetação na fase de plântulas. Os demais genótipos, embora mais suscetíveis, diferenciaram-se quanto ao comportamento à doença, podendo ser classificados quanto ao tipo de infecção de intermediários. Não foi observado genótipo suscetível, no nível de reação tipo 4 . Constatou-se que, em muitos genótipos, houve variação na suscetibilidade para determinada raça patogênica inoculada, expressa por uma ou mais plântulas suscetíveis.

Tabela 4. Produtividade média $\left(\mathrm{kg} \mathrm{ha}^{-1}\right)$ de grãos dos genótipos de trigo avaliados em condição de sequeiro, nas localidades de Manduri (Zona A), Capão Bonito e Itapeva (Zona B) e em Paranapanema (Zona C), no período de 2003 a 2005, no Estado de São Paulo

\begin{tabular}{|c|c|c|c|c|c|}
\hline Genótipos & 2003 & 2004 & 2005 & Média & $\%$ \\
\hline & \multicolumn{5}{|c|}{$\longrightarrow \mathrm{kg} \mathrm{ha}^{-1}$} \\
\hline 1- BH1146/ / AA"S" / WIN"S" /3/IAC 227 & 2916 ae & 3112 a-d & $2297 \mathrm{bd}$ & 2801 a-d & 98,59 \\
\hline 2- BH1146/ / AA"S" / WIN"S" /3/IAC 227 & $3061 \mathrm{ad}$ & $2672 \mathrm{e}-\mathrm{g}$ & 2762 a & 2831 a-d & 99,65 \\
\hline \multicolumn{6}{|l|}{ 3- BH1146//AA"S" /WIN"S"/6/IAS58/4/KAL/BB/CS"S"/3/ALD } \\
\hline "S" / 5/BOW"S" & $2515 \mathrm{fg}$ & $2394 \mathrm{~g}-\mathrm{h}$ & 2147 de & $2352 \mathrm{~g}-\mathrm{h}$ & 82,79 \\
\hline 4- BH1146/ / AA"S" / WIN"S" /3/IAC 24 & $2751 \mathrm{df}$ & $2818 \mathrm{~d}-\mathrm{f}$ & $2396 \mathrm{bd}$ & 2655 d-e & 93,45 \\
\hline 5- BH1146/ / AA"S" /WIN"S" /3/IAC 24 & $3159 \mathrm{ab}$ & $3052 \mathrm{~b}-\mathrm{d}$ & $2427 \mathrm{bd}$ & 2879 a-c & 101,34 \\
\hline 6- BH1146//AA"S"/WIN"S"/3/TUI"S" & $2663 \mathrm{eg}$ & $2545 \mathrm{f}-\mathrm{h}$ & 2482 ac & 2563 e-f & 90,21 \\
\hline 7- BH1146//AA"S"/WIN"S"/3/HAHN*2/PRL & $3112 \mathrm{ad}$ & $3073 \mathrm{~b}-\mathrm{d}$ & 2508 ac & 2920 a & 102,78 \\
\hline 8- BH1146//AA"S" /WIN"S" /3/BUC/FKL/ / MYNA/VUL & $3158 \mathrm{ab}$ & $3084 \mathrm{~b}-\mathrm{d}$ & $2548 \mathrm{ab}$ & 2930 a & 103,13 \\
\hline 9- BH1146// AA"S" / WIN"S" / 4/INIA/A.DIST//INIA/3/GEN & 2970 ae & 2957 c-e & $2417 \mathrm{bd}$ & $2781 \mathrm{a}-\mathrm{d}$ & 97,89 \\
\hline 10- IAC 1004 & 3278 a & $3329 a-b$ & $1844 \mathrm{f}$ & 2817 a-d & 100,00 \\
\hline 11- BH1146// AA"S" / WIN"S" /3/SERI 82 & $2837 \mathrm{bf}$ & 3433 a & $2323 \mathrm{bd}$ & 2864 a-d & 100,81 \\
\hline 12- BH1146// AA"S" /WIN"S"/3/VEE//DOVE/BUC & $2775 \mathrm{cf}$ & $2355 \mathrm{~g}-\mathrm{h}$ & $2359 \mathrm{bd}$ & $2496 \mathrm{f}-\mathrm{g}$ & 87,86 \\
\hline 13- BH1146//AA"S" /WIN"S" /3/CNO79/PRL & 2912 ae & 2976 c-e & $2436 \mathrm{bd}$ & 2774 a-d & 97,64 \\
\hline 14- BH1146// AA"S" /WIN"S"/3/VEE//DOVE/BUC & $3193 \mathrm{ab}$ & $2517 \mathrm{f}-\mathrm{h}$ & $2396 \mathrm{bd}$ & 2702 c-e & 95,11 \\
\hline 15-IAC 289 & 3136 ac & 2928 c-e & 2533 ac & 2865 a-d & 100,00 \\
\hline 16-KAUZ/3/BH 1146/ / AA"S" / WIN"S" & $2363 \mathrm{~g}$ & $2265 \mathrm{~h}$ & 1966 ef & $2198 \mathrm{~h}$ & 77,37 \\
\hline 17-VEE/ / DOVE/BUC/3/BH1146// AA"S" / WIN"S" & 2895 be & 3258 a-c & $2510 \mathrm{ac}$ & $2887 \mathrm{a}-\mathrm{c}$ & 101,62 \\
\hline 18-CMH 75.A.66/SERI/3/BH1146/ / AA"S" /WIN"S" & 3133 ac & 3160 a-d & 2492 ac & 2928 a & 103,06 \\
\hline 19-BH1146//AA"S" /WIN"S" /3/TAN/PEW//SARA & 2832 bf & $3080 \mathrm{~b}-\mathrm{d}$ & $2235 \mathrm{~cd}$ & 2715 b-e & 95,56 \\
\hline 20- BH1146//AA"S" /WIN"S" / 4/RL6010/2*YR/3/2*VEE"S" / GEN & $3031 \mathrm{ad}$ & 3174 a-c & 2503 ac & $2902 a-b$ & 102,15 \\
\hline Média & 2934 A & $2922 \mathrm{~A}$ & $2378 \mathrm{~B}$ & 2745 & - \\
\hline Anos & - & - & - & $185,78^{* *}$ & - \\
\hline Genótipo & $4,73 * *$ & $10,44^{* *}$ & $5,35^{* *}$ & $11,14^{* *}$ & - \\
\hline Local & $575,23 * *$ & $99,92 * *$ & $301,07^{* *}$ & $196,96 * *$ & - \\
\hline Ano $x$ Local & - & - & - & $396,19 * *$ & - \\
\hline Ano x Genótipo & - & - & - & $4,47^{* *}$ & - \\
\hline Genótipo x local & $2,79 * *$ & $4,10^{* *}$ & $3,47^{* *}$ & $5,31^{* *}$ & - \\
\hline Ano x Local x Genótipo & - & - & - & $2,37^{* *}$ & - \\
\hline $\mathrm{CV} \%$ & 14,93 & 14,47 & 15,10 & 15,18 & - \\
\hline
\end{tabular}

Médias para comparação da produtividade de grãos entre cultivares dentro de ano e na média geral em letras minúsculas para comparação entre anos em letras maiúsculas. Médias seguidas por letras distintas diferem entre si pelo teste de Duncan a $5 \% .{ }^{*} ;{ }^{* *}$ significativo a $5 \%$ e $1 \%$ respectivamente.

\% em relação as média das testemunhas IAC 1004 e IAC $289\left(2841\right.$ kg.ha $\left.{ }^{-1}\right)$. 
Tem se verificado que muitos fatores são limitantes ao rendimento ou ao potencial produtivo de uma cultivar, entretanto, fatores limitantes em uma região podem não ser significativos em outra. A adaptação ampla, o potencial de rendimento e a resistência às doenças são fatores inerentes de uma base genética ampla, no processo de melhoramento genético.

Pelas análises estatísticas dos experimentos, por ano, constataram efeitos altamente significativos para genótipo, local e para a interação genótipo x local. A análise conjunta também demonstrou efeito altamente significativo para ano, local, genótipo e para as interações ano $x$ local, ano $x$ genótipo, genótipo $x$ local e ano $\times$ local $\times$ genótipo, de acordo com a tabela 4 . Observou-se, que os efeitos do fator ano influenciaram sobre o potencial produtivo dos genótipos, o que revela comportamento diferencial dos genótipos em relação o ambiente ano. O rendimento da cultivar IAC 1004 de Triticum durum foi relevante em 2003 e 2004, entretanto, ocorreu baixo rendimento em 2005, devido à baixa precipitação pluvial no período.
Os genótipos 8(BH1146// AA"S" / WIN"S" / 3/BUC/FKL//MYNA/VUL), 7 (BH1146// AA"S" / WIN"S"/3/HANN*2/PRL) e 18 (CMH 75.A.66/ SERI/3/BH1146//AA"S"/WIN"S") proporcionaram os melhores rendimentos de grãos no período. O menor rendimento foi obtido pelo genótipo 16 (KAUZ/ 3/BH1146// AA"S"/WIN"S".

Como a análise de variância conjunta, em relação a rendimento de grãos, houve interação genótipo $\mathrm{x}$ anos, foi possível realizar a análise da estabilidade e da adaptabilidade, conforme a tabela 5 para o triênio 2003 a 2005, que revelou comportamento diferencial entre os genótipos para o fator (ambiente) ano, expresso nos valores de $b_{1}$ muito contrastantes para os genótipos estudados. O IAC 1004 apresentou-se altamente responsivo (b $=2,68^{* *}$ ) representando padrão de resposta para ambiente favorável, mas com respostas negativa nos ambientes desfavoráveis, sendo com baixa estabilidade. Os genótipos 2, 6 e 12 expressaram seu potencial de rendimento em ambiente desfavorável.

Tabela 5. Estabilidade e adaptabilidade dos genótipos de trigo avaliados em condição de sequeiro, no período de 2003 a 2005 nas localidades de Manduri (Zona A), Capão Bonito e Itapeva (Zona B) e em Paranapanema (Zona C), no Estado de São Paulo

\begin{tabular}{|c|c|c|c|c|}
\hline Genótipos & Média & $\mathrm{b}=1^{(1)}$ & Sd (2) & $\mathrm{R}^{2}$ \\
\hline & $\mathrm{kg} \mathrm{ha}^{-1}$ & & $\mathrm{~kg} \mathrm{ha}^{-1}$ & $\%$ \\
\hline 1- BH1146//AA"S" /WIN"S" /3/IAC 227 & $2775 \mathrm{a}$ & 1.30 & $119 \mathrm{~ns}$ & 92,73 \\
\hline 2- BH1146/ / AA"S" /WIN"S" /3/IAC 227 & 2831 a & $0.21^{* *}$ & $248^{*}$ & 11,20 \\
\hline \multicolumn{5}{|l|}{ 3- BH1146//AA"S" /WIN"S" /6/IAS58/4/KAL/BB/CS"S" /3/ALD } \\
\hline "S" / 5/BOW"S" & $2352 \mathrm{bc}$ & 0.57 & $-(3)$ & 91,93 \\
\hline 4- BH1146/ / AA"S" / WIN"S" / 3/IAC 24 & $2655 \mathrm{ab}$ & 0.71 & - & 96,48 \\
\hline 5- BH1146/ / AA"S" /WIN"S" /3/IAC 24 & 2879 a & 1.25 & - & 99,09 \\
\hline 6- BH1146/ / AA"S" /WIN"S"/3/TUI"S" & $2563 \mathrm{ac}$ & $0.23^{* *}$ & - & 62,72 \\
\hline 7- BH1146//AA"S" /WIN"S" /3/HAHN*2/PRL & 2897 a & 1.07 & - & 99,97 \\
\hline 8- BH1146//AA"S" / WIN"S" /3/BUC/FKL/ / MYNA/VUL & 2930 a & 1.05 & - & 99,49 \\
\hline 9- BH1146//AA"S" / WIN"S" / 4/INIA/A.DIST//INIA/3/GEN & 2781 a & 1.06 & - & 99,96 \\
\hline 10- IAC 1004 & 2817 a & $2.68^{* *}$ & - & 99,50 \\
\hline 11- BH1146/ / AA"S" / WIN"S" /3/SERI 82 & $2864 \mathrm{a}$ & 1.45 & $434^{* *}$ & 67,48 \\
\hline 12- BH1146// AA"S" /WIN"S"/3/VEE//DOVE/BUC & 2496 ac & $0.40^{*}$ & $268^{*}$ & 27,84 \\
\hline 13- BH1146/ / AA"S" /WIN"S" /3/CNO79/PRL & 2774 a & 0.93 & - & 97,79 \\
\hline 14- BH1146// AA"S" /WIN"S"/3/VEE//DOVE/BUC & $2702 \mathrm{ab}$ & 0.88 & $449 * *$ & 42,03 \\
\hline 15-IAC 289 & $2865 \mathrm{a}$ & 0.93 & $70 \mathrm{~ns}$ & 90,93 \\
\hline 16-KAUZ/3/BH 1146/ / AA"S" / WIN"S" & 2198 c & 0.64 & - & 96,10 \\
\hline 17-VEE//DOVE/BUC/3/BH1146// AA"S" /WIN"S" & $2887 \mathrm{a}$ & 1.01 & $252^{*}$ & 72,93 \\
\hline 18-CMH 75.A.66/SERI/3/BH1146// AA"S" / WIN"S" & 2928 a & 1.20 & - & 99,42 \\
\hline 19- BH1146// AA"S" /WIN"S" /3/TAN/PEW//SARA & $2715 \mathrm{ab}$ & 1.31 & $166 \mathrm{~ns}$ & 89,49 \\
\hline 20- BH1146/ / AA"S" /WIN"S" / 4/RL6010/2*YR/3/2*VEE"S" / GEN & 2902 a & 1.09 & $51 \mathrm{~ns}$ & 94,15 \\
\hline
\end{tabular}

Médias para comparação da produtividade de grãos entre cultivares em letras minúsculas diferem entre si pelo teste de Duncan a $5 \%$;

$\left({ }^{1}\right)$ Coeficiente de regressão linear. * $\mathrm{e}{ }^{* *}$ significativo a 5 e a $1 \%$ pelo teste de $\mathrm{t} ;{ }^{2}$ ) Desvio de regressão, ${ }^{*} \mathrm{e}{ }^{* *}$ significativo a 5 e a $1 \%$ pelo teste de F.; $\left({ }^{3}\right)$ Variância negativa do erro e ns = não significativo. 
Os genótipos 2 (BH1146//AA"S" / WIN"S" / 3/IAC 227) R ${ }^{2}=11,20,6$ (BH1146// AA"S" / WIN"S" / 3/TUI"S") $R^{2}=6,62,12$ (BH1146//AA"S"/WIN"S"/ 3/VEE// DOVE/BUC) $R^{2}=27,84$ e $14 R^{2}=42,03$ proporcionaram baixa previsibilidade de comportamento, com coeficiente de determinação inferior aos demais genótipos. O genótipo 16 (KAUZ/ 3/BH 1146//AA"S" /WIN"S") com rendimento de grãos inferior aos demais genótipos não deve ser julgado como indesejável, uma vez que seu $\mathrm{R}^{2}$ atingiu níveis de $90,1 \%$, provavelmente, seu ciclo mais longo, deva ter contribuído para esta baixa adaptação regional.

De acordo com Baethgen (1997), a correlação anos e ambiente (local ou ano) em que se obtém altos rendimentos de grãos geralmente se caracterizam por uma combinação muito favorável de quase todos os fatores que afetam o rendimento, portanto, e impossível de se quantificar impactos relativos de cada fator envolvido no elo da cadeia de produção.

\section{CONCLUSÕES}

1. O emprego da linhagem BH1146//AA"S" / WIN"S", oriunda de cruzamento interespecífico ( $T$. aestivum $\times$ T. durum) e irradiação gama, foi bastante eficiente na obtenção de novos genótipos de trigo com resistência ao agente causal da ferrugem da folha associada a bons rendimentos de grãos.

2. O genótipo 8 (BH1146//AA"S" /WIN"S" / 3/BUC/FKL//MYNA/VUL) foi resistente à ferrugem da folha nos testes em plântulas; no campo, a cultivar IAC 1004 foi imune à doença em condições de infecção natural no campo.

3. Os melhores rendimentos de grãos foram obtidos pelos genótipos 8 (BH1146// AA"S" / WIN"S"/3/BUC/FKL//MYNA/VUL), 7 (BH1146/ / $\left.\mathrm{AA}^{\prime \prime} \mathrm{S}^{\prime} / \mathrm{WIN}^{\prime \prime} \mathrm{S}^{\prime \prime} / 3 / \mathrm{HANN}^{*} 2 / \mathrm{PRL}\right)$ e 18 (CMH 75.A.66/SERI/3/BH1146//AA"S"/WIN"S").

4. Como as reações dos genótipos foram diferenciadas para as diferentes raças de ferrugem da folha, portanto, caracterizando diferentes fontes de resistência à doença, os mesmos podem ser utilizados em programas de melhoramento genético como fonte de resistência para essa doença.

\section{AGRADECIMENTOS}

Os autores agradecem aos técnicos de apoio Carlos Aparecido Fernandes, José Norberto da Silva e José Angelino de Paula pela colaboração no desenvolvimento dos experimentos.

\section{REFERÊNCIAS}

ANGRA, D.C., MARTINELli, P., PRESTES, A.M. \& FERNANDES, M.I.B. Haplodiploidização de híbridos intergenéricos via gimnogênese. Brazilian Journal of Genetics, Ribeirão Preto, v.19 (Supl), n.3, p.124, 1996. (Resumo)

BAETHGEN, W.E. Potenciales de rendimiento de trigo. In KOHLI,M.M. y MARTINO, D.L. (Ed.). Explorando Altos Rendimentos de Trigo. La Estanzuela, Uruguay: CIMMYTYINIA, 1998. p.127-148.

BARBOSA, M.M., PRESTES, A.M.; ANGRA, D.C. Agropyron como fonte de resistência à mancha bronzeada do trigo. Fitopatologia Brasileira, Brasília, v.18, p.335-347, 1993.

BARCELLOS,-A. L. Ferrugem da folha do trigo no Brasil. População patogênica, fontes de resistência, trigos comerciais, perpetuação e controle quimico. In: MOLESTINA, C.J. (Ed.). Diálogo. REUNIÓN DE ESPECIALISTA EM ROYAS DE CEREALES DE INVIERNO, 13., 1986. Montevideo, Uruguay, 1986. p.73-87.

BARCELLOS, A. L. Populações patogênicas de Puccinia triticina (Ferrugem da folha do trigo) no período de 1997-1999. In: Reunião Nacional de Pesquisa de Trigo, 18., 1999, Passo Fundo. Resumos... Passo Fundo: CNPT, 1999. v.2, p.524-528.

CAMARGO, C.E.O.; FELICIO, J.C.; TULMANN NETO, A.; FERREIRA FILHO, A.W.P.; PETTINELLI JUNIOR, A.; CASTRO, J.L. Melhoramento do trigo: XXVIII. Novos genótipos obtidos por seleções em população segregante interespecífica submetida à irradiação gama. Bragantia, Campinas, v. 54, n.1, p. 51-65, 1995.

CAMPINAS, INSTITUTO AGRONÔMICO Recomendações Técnicas de Trigo para 2002. 3.ed.atual. Campinas, 2002. 92p.

CHAVES, M. S.; BARCELLOS, A. L.; GERMÁN, S. ;SCHEEREN, P. L. ; DEL DUCA, L.J.A. ; SÓ-SILVA, M.; CAIERÃO, E. Population dynamics of Puccinia triticina in the South Cone region of South America from 1997 to 2004. In: INTERNATIONAL WHEAT CONFERENCE, $7^{\text {th }}, 2005$, Mar del Plata. Abstracts... Mar del Plata: SAGPyA/INTA, 2005. p.130.

CUNHA, G. R. Buscando a elevação do rendimento de grãos em trigo. Passo Fundo: Embrapa Trigo, 2005. 7p. (Embrapa Trigo. Documentos Online, 50). Disponível em: http:// www.cnpt.embrapa.br/biblio/do/p_do50.htm

EBERHART, S.A.; RUSSELL,W.A. Stability parameters for comparing varieties. Crop Science, Madison, v.6, p.36-40, 1966.

FELICIO, J.C.; CAMARGO, C. E. O.; CASTRO, J.L.; GERMANI, Rendimento de grãos e sua relação com as doenças e variáveis climáticas em Capão Bonito de 1994 a 2001. Bragantia, Campinas, v. 63, n.1, p. 93-103, 2004.

GERMAN, S.; KOHLI, M.M. ; CHAVES, M.S. ; BARCELLOS, A. ; NISI, J. ; ANNONE, J. ; MADARIAGA, R.; VIEDMA, L. Breakdown of resistance of wheat cultivars and estimated losses caused by recent changes in the leaf rust population in South America. In: INTERNATIONAL CEREAL RUSTS 11., \& 
POWDERY MILDEWS CONFERENCE, 2004, Norwich. Abstracts.... Norwich : John Innes Centre, 2004. part 2-s.A2, p. 21-21.

LARGE, E.C. Growth stages in cereals. Illustration of the Feekes Scale. Plant Pathology, London, v.3, p.128-129, 1954.

LONG, D. L.; KOLMER, J. A. A North American system of nomenclaturefor Puccinia recondita f. sp. tritici. Phytopathology, v. 79, p. 525-529. 1989.

NELSON, R.R.; Introduction. In: NELSON, R.R. (Ed.). Breeding plants for disease resistance. Pensiylvania: The Pennsylvania State University Press, 1973. p.3-12.

PICININI, E.C.; FERNANDES, J.M.C. Controle das doenças de trigo. In CUNHA, G.R.; BACALTCHUK, B. (Eds.). Tecnologia para produzir trigo no Rio Grande do Sul, Porto Alegre: Assembléia Legislativa, Comissão de Agricultura, Pecuária e Cooperativismo. Passo Fundo: Embrapa Trigo, 2000. p.225-243. (Série Culturas, n. 02).

PIMENTEL-GOMES, F. Curso de estatística experimental 4.ed.ver.ampl. Piracicaba: Nobel, 1970. 430p.

PRIESTLEY, R.H.; BAYLES, R.A. The contribution and value of resistant cultivars to disease control in cereals. In: CLIFFORD, B.C.; LESTER, E. (Ed.). Control of Plant Disease: costs and benefits. Oxford: Blackwell Scientific Publications, 1988. p.5365.

RAIJ, B. van; CANTARELLA, H.; QUAGGIO, J.A.; FURLANI, A..M. Recomendações de Adubação e calagem para o Estado de São Paulo. Campinas: Instituto Agronômico/ Fundação IAC, 1996. 285p. (Boletim técnico 100)

REIS, E.M.; CASA, R.T.; FORCELINI, C.A. Relação entre a severidade e a incidência da ferrugem da folha do trigo, causada por Puccinia recondita f. sp. tritici. Fitopatologia Brasileira, Brasília, v.21, p.369-372, 1996.

ROELFS. A.P.; SINGH, R.P.; SAARI, E.E. Rust Diseases of Wheat: concepts and methods of disease management. Mexico, D. F.: CIMMYT, 1992. 81p.

SCHRAMM, W.; FULCO, W.S.; SOARES, M.H.G.; ALMEIDA, A.M.P. Resistência de cultivares de trigo em experimentos ou cultivo no Rio Grande do Sul, às principais doenças fúngicas. Agronomia Sulriograndense, Porto Alegre, v.10, p.31-39, 1974.

SOUSA, C.N.A. Contribuição das cultivares de trigo de Strampelli para o melhoramento de trigo no Brasil. Passo Fundo: Embrapa Trigo, 2001. 5p. (Embrapa Trigo. (Documentos Online, 7). Disponível em: http://www.cnpt.embrapa.br/biblio/ p_do07.htm 